\title{
The philosophy of Big Life
}

\author{
Ruiyu Zhu*1 \\ *Jiangnan University, Wuxi, P.R. China.
}

\begin{abstract}
This article introduces the main points of the Big Life philosophy. First, the exposition of a scientific interpretation of Yin-Yang and Wu Xing (the Five Elements theory) provides a connection between Eastern and Western philosophy and illuminates the underlying laws of the complex system. Second, there is a presentation of the ubiquitous natural driving force embedded in our objective material world. A comprehension of the natural driving force is the basis for an understanding of the evolutionary trend of nature and its integration with nature. Third, an explanation of the underlying nature of life is offered, with an emphasis on the need for a deeper interpretation of life to expand its possibilities, remove constraints, and achieve true freedom. Fourth, there is a discussion of the composition and characteristics of the conscious environment. The self in the conscious environment is the true self. Therefore, the construction of the conscious environment is the most important work of human beings. Finally, there is a section on the rational reconstruction of the spiritual world. The most pressing problems currently faced by society and individuals are rooted in the spiritual world that has yet to be reconstructed. The paper concludes with a proposal to establish life values with the Objective Belief of Nature as the concept central to that pursuit.
\end{abstract}

\section{Introduction}

The primary logic that rules in today's world is based on the modern Western cognitive system. This logic determines the shape of political, economic, and cultural systems and expressions in most of the world's countries. Moreover, it affects the consciousness, cognition. and values of most people. Obviously, the current state of contradictions and conflicts show that there are many defects in this structure. Although, on the surface, such a structure may seem rational and logical, it is guided by a contradictory need for satisfaction and corresponds to a spirit crammed between a body's desire and a cultural void with regard to inner growth.

Underlying the political, economic, and cultural framework of this pursuit to satisfy needs in today's world, personal desires continue to expand; the inner soul gradually is lost. The increasingly serious environmental crisis is accompanied by worsening

\footnotetext{
${ }^{1}$ This is a working document which has not been peer reviewed yet. Please address any comment or remark to ry_zhu@hotmail.com or ry_zhu@sina.com.
} 
geopolitical conflicts. Consequently, the entire world becomes increasingly unstable in the manifestation of one-sided desires. We constantly are immersed in a culture of satisfaction and gradually lose the vitality of life. The philosophy that represents the highest human cognition has been discarded by people in dark places; it no longer is able to provide guidance for various disciplines as it cannot shine its light. Various disciplines are producing a growing amount of noise, rather than true knowledge. Consequently, people increasingly are confused about the world. What kind of science should our science be? What kind of education should our education be? What kind of culture should our culture be? What kind of economy should our economy be? What kind of civilization should our civilization be? In addition, how do we genuinely permit those people, social groups and countries who are in the early stages of the understanding of material laws and are trapped in that space between the pursuit of satisfaction and the absence of inner growth, emerge from material poverty? How do we truly allow those who are in the early stage of the understanding of spiritual laws and fall into that trap escape this spiritual dilemma? How do we free those who have been kidnapped by desire and have lost their souls and help them regain balance in their lives and blossom in the glory of their lives?

After many decades of deep thinking, I discovered a true answer to these questions and put forward the philosophy of Big Life. The philosophy of Big Life is based on the reinterpretation of Chinese Yin-Yang philosophy, Western science, complex systems, the essence of consciousness, and the essence of life. It represents a means to systematically reorganize the cognition of the world in its essence and put forth a set of rational solutions. The purpose is to make satisfaction-driven consciousness, culture, economy and politics move towards vitality-based consciousness, culture, economy, and politics. The objective is to build a human civilization full of vitality. In fact, all problems are ultimately attributed to the lack of vitality, and they are related to an incomplete understanding of life. The philosophy of Big Life focuses on the most basic laws of natural life, allows everyone's true soul to blossom from the depths of the human heart, and permits people to truly love themselves. The improvement of personal cognition and spiritual vitality gradually will lead to a solution for all problems of human society. The objective is to dig down to the very roots - the extremely strong driving force of nature-so that people's actions may return to the process of natural evolution, their souls may return to the pulse of natural movement, and they may let their own beauty flourish. Together humans can support the evolution of human society to a higher level. Only in this way, all problems will no longer be problems but merely steps to be taken in the process of growth. Eastern and Western cultures will blend into each other and become the two poles of human society's development, ultimately forming a common and beautiful bloom of human civilization. The philosophy of Big Life has discovered the first driving force derived from natural evolution that can make people and human society develop in beautiful ways.

The main components of the philosophy of Big Life include the scientific connotation of Yin-Yang and $\mathrm{Wu}$ Xing, the composition and characteristics of consciousness, the natural driving force, an underlying understanding of life, and the active reconstruction of the spiritual world. What follows is only a partial description of the philosophy of Big Life. The Big Life philosophy also will serve to reorganize existing cultures and science, rebuild a modern philosophical system, and return the resulting philosophical system to the center of various disciplines to guide the actions of humans and the direction of society. The goal is to integrate human life to the 
evolutionary trend of nature, so that the development of human society and personal development may return to their roots. Life as referred to in the Big Life philosophy is the awareness of the essence of basic life and its movement integrated with nature; life may cover all complex systems of movement as well as all complex phenomena, including human beings. The Big Life philosophy respects and recognizes all existing forms of faith. It will become the most basic scientific explanation for all types of beliefs. The Big Life philosophy focuses on change from the inside to the outside. The philosophy conforms to the driving force of nature and focuses on the natural solution to problems from the inside out. Therefore, there is a respect for-and no negation of - any form of representation.

The various viewpoints described represent preliminary versions. There are several reasons for which objective conditions have made it difficult for me to completely calm down and further improve upon these reflections. My aim is to have these thoughts published, with the hope that more people will participate and we may jointly improve, practice together, and encourage the vitality of individuals and the blooming of society.

\section{Brief introduction to each section ${ }^{2}$}

\section{Scientific Connotation of the Five Elements Theory}

The Five Elements theory (Wuxing) is the philosophical foundation of traditional Chinese medicine and culture. 'Metal', 'Water', 'Wood', 'Fire', and 'Earth' are the five basic elements of the Five Elements theory. However, due to its complexity, people's understanding of the theory has remained superficial, giving rise to great differences in understanding. Scientists often regard the Five Elements theory as unscientific. At the same time, enthusiasts of traditional Chinese culture often overuse it and reach specious conclusions, posing a challenge to rational understanding by the public. This study proposes that in nature-a dynamic and complex system-evolution determines the existence of the law of matter, and the law of matter exists to better perform evolution. Yin-Yang and Wuxing are the laws of evolution. Using scientific logic, it is also shown that Wuxing is the most basic movement mode of complex dynamic systems, reflecting the basic dynamic mechanism of complex systems' responses to environmental information. This study further proposes that 'Metal' is the control center of the entire system and represents the response, processing, and distribution of internal information commands. 'Water' represents the process of internalizing energy and the inflow of basic materials. 'Wood' represents the process of forming a high-level substance based on information. 'Fire' represents the externalization of energy and the realization of the function of advanced matter. Finally, 'Earth' represents the process of returning advanced substances to basic substances. Revealing the scientific nature of the Five Elements can provide new ideas for the human cognition of complex phenomena and provide a new perspective for solving complex system problems.

\footnotetext{
${ }^{2}$ In order to facilitate communication and highlight each viewpoint, the details of each viewpoint are in different articles, and only its summary is listed here.
} 


\section{The composition and characteristics of conscious environment}

Although we are faced with a complicated and objective world, the conscious environment is the world that the spiritual self can directly perceive; the real self lives in the conscious environment. This artical discusses the composition and characteristics of human conscious environment. First, this article proposes that the conscious environment is composed of five aspects: conscious scene, experience circuit, emotional label, concept, and values. Second, it provides a discussion of the thinking, reasoning, beliefs, and the theory of use and disuse upon which the further growth of cognition depends. Finally, it explains other characteristics of cognition. For example, knowing determines perception, the easily imprisoned soul, its assimilation, and the limitations of one's own cognition. Moreover, the importance of emptying is recognized. This part of the article presents the concept of conscious environment for the first time and systematically discusses the influencing factors and characteristics of the human conscious world. Its purpose is to provide a preliminary and systematic understanding of the human spiritual world and to provide a theoretical basis for the following concepts: the natural driving force, the underlying thinking about life, and the rational reconstruction of the spiritual world.

\section{Natural driving force}

We seem to be able to think about everything and imagine everything. However, we are just a higher form of natural evolution and cannot escape the shackles of nature. Our world is moving and changing. We inevitably feel that a force beyond our control drives everything and pushes everything forward. This artical discusses the natural driving force hidden in the objective world and the human body. There are five basic points: 1) Natural driving forces are everywhere and control everything; 2) the natural driving force from the inside out; 3) expectations about the outcome of human-related matters, determine the choice of cause; 4) the natural driving force for society moves from individual to society and therefore only a full realization of everyone's life will produce a better society; and, 5) the foundation of life's growth is to face reality and pain by acknowledging the driving forces.

\section{Understanding the essence of life}

What is the essence of life? Everyone may have a different answers in their hearts. However, is there a most basic answer to the nature of life? To respond, it is necessary to be able to remove everything at the material level and place it into the process of natural evolution. In this article, I present a systematic analysis of the root of life for the first time. First, I propose that the movement form of life is a layer-by-layer iteration of "change and unchanged." Moreover, the existing form of life is a periodic cycle of five elements. Second, initiative is the first driving force of life. Whether for individuals or society, we need to be able to actively construct in order to truly unleash the vitality of our lives. Finally, human life lies in the construction of a conscious environment full of vitality. Human life for society resides in the ability to encourage of more human lives to bloom. Although it seems that one should be able to take the initiative to control everything, happiness cannot be separated from a material base. Humans are merely a more advanced form of natural evolution-a form that is able to actively regulate natural evolution. The most essential truth of life 
described here is the truth consistent with the driving force of nature. Therefore, the essence of life described here can help everyone's life grow and bloom.

\section{Active reconstruction of the spiritual world}

The spiritual world is constrained passively by primitive urges. Similar to an uncultivated wasteland, it will necessarily breed messy plants. Most of the time we are imprisoned by the social environment, a narrow understanding of ourselves, and our own desires. This makes it difficult for life itself to truly shine and enjoy authentic vitality. People are the foundation of society. The fact that individuals are unable to rebuild the spiritual world makes it difficult for human society to achieve true growth. It is difficult for people to obtain real happiness and it is difficult for the rulers to achieve their long-cherished wishes. This article first presents an argument for why reconstruction is needed, accompanied by a proposal that faith is the basis for reconstruction. Additionally, I propose the concept of the Objective Belief of Nature. The crucial component of this belief is the need to pay less attention to ourselves and use love together with the laws of nature to promote the beautiful development of society. The purpose is to make people truly love themselves, so that they may enjoy vitality in their lives and achieve happiness (in the passive sense of happiness). Similarly, society has vitality because of the blossoming of everyone's life. Likewise, a society filled with vitality further promotes the blooming of everyone's life. Such a belief provides ideological and theoretical support for humankind's further development. The Objective Belief of Nature mainly emphasizes a belief; it does not reject or privilege any given form.

\section{Other Thoughts on the Philosophy of Big life}

This artical supplements the information that the Big Life philosophy fails to adequately explain. First, there is a discussion of the relationship between the five basic views of the Big Life philosophy. The point is made that these five basic views are consistent with the five basic elements of the five-element system elaborated from the systematic laws of life present in the material world. Second, it analyzes the main factors that affect human cognition, which include the following: the complexity of things, the characteristics of the cognitive path, factors related to the observer, and environmental factors. It is precisely because of these natural problems that we have direction and goals for growth in our lives. The process of continuously solving these problems is also the process of continuous growth and growing prosperity. Finally, five basic levels of the spiritual world are proposed. There is an emphasis on the transition from the rational world to the constructed world as an urgent task for human beings. This offers a basis for an integration with natural evolution that will promote humans' true vitality.

\section{References}

1. zhu, ruiyu. "Scientific Connotation of the Five Elements Theory." SocArXiv, 19 June 2020. Web.

2. zhu, ruiyu. "The Composition and Characteristics of Conscious Environment." OSF Preprints, 20 July 2020. Web.

3. zhu, ruiyu. "Natural Driving Force." OSF Preprints, 20 July 2020. Web. 
4. zhu, ruiyu. "Understanding the Essence of Life." SocArXiv, n.d. Web.

5. zhu, ruiyu. "Active Reconstruction of the Spiritual World." OSF Preprints, 20 July 2020. Web.

6. zhu, ruiyu. "Other Thoughts on the Philosophy of Big Life." SocArXiv, n.d. Web. 